Rabaska

Revue d'ethnologie de l'Amérique française

\title{
Histoires de pêche
}

\section{René Bouchard}

Volume 18, 2020

URI : https://id.erudit.org/iderudit/1072952ar

DOI : https://doi.org/10.7202/1072952ar

Aller au sommaire du numéro

Éditeur(s)

Société québécoise d'ethnologie

ISSN

1703-7433 (imprimé)

1916-7350 (numérique)

Découvrir la revue

Citer ce compte rendu

Bouchard, R. (2020). Compte rendu de [Histoires de pêche]. Rabaska, 18, 422-425. https://doi.org/10.7202/1072952ar

Ce document est protégé par la loi sur le droit d'auteur. L'utilisation des services d'Érudit (y compris la reproduction) est assujettie à sa politique d'utilisation que vous pouvez consulter en ligne.

https://apropos.erudit.org/fr/usagers/politique-dutilisation/
Cet article est diffusé et préservé par Érudit.

Érudit est un consortium interuniversitaire sans but lucratif composé de l'Université de Montréal, l'Université Laval et l'Université du Québec à Montréal. Il a pour mission la promotion et la valorisation de la recherche. https://www.erudit.org/fr/ 


\title{
Histoires de pêche
}

\author{
RENÉ BOUCHARD \\ Société québécoise d'ethnologie
}

Voilà une exposition superbement montée et qui met en valeur pour la première fois la richesse de l'imposante collection du Musée de la civilisation (McQ) dans le domaine de la pêche, la plus importante en nombre et en qualité au Québec, et certainement l'une des plus remarquables au Canada. Son titre, Histoires de pêche, au pluriel, a l'ambition de raconter aussi bien la grande histoire de ce sport que les multiples petites histoires particulières qui émaillent le discours de tout pêcheur passionné par la pratique de son sport favori. Sa présentation s'alimente en contenus aux meilleures sources qui soient, en particulier sur les recherches compétentes menées sur le sujet depuis une quarantaine d'années par l'ethnologue et historien Paul-Louis Martin (voir dans le présent numéro de la revue son article synthèse sur L'histoire de la pêche sportive au Québec). Bref, une exposition qui a du «panache » et qui n'a surtout pas l'air de prendre «l'eau»!

Le discours narratif de l'exposition repose sur la présentation de quatre thèmes majeurs : le Québec, paradis de la pêche ; le lac virtuel ; les types de pêche ; et leurs à-côtés. D'entrée de jeu, le visiteur est saisi par la beauté des bannières publicitaires des compagnies de transport ferroviaire ou maritime (Pacifique Canadien, Grand Tronc, etc.), des revues ou guides spécialisés (Canadian Sportsman, Forest Outdoors, Sports Afield) et du gouvernement qui vantent, dès la fin du XIX ${ }^{e}$ siècle, le Québec comme un très vaste territoire sauvage et giboyeux rendu enfin accessible aux pêcheurs. À cette période où les gens ordinaires n'ont que le dimanche pour se reposer, de telles publicités s'adressent surtout à des voyageurs aisés des classes supérieures de la société. Chemins de fer et haute finance vont en effet de pair dans la pénétration de l'hinterland québécois et la promotion d'une économie touristique. La découverte de lacs poissonneux s'adresse dans ces années-là plutôt aux sportifs qui ont les moyens de se payer, pour leur profit parfois exclusif, quelques semaines d'évasion en pleine nature sauvage. L'accès aux paysages de la Mauricie, du Saguenay ou de la Gaspésie se fait l'écho de la quête paradisiaque du grand air qui entraîne dans son sillage nombre de pêcheurs relatant leurs aventures dans plusieurs publications diffusées au Canada et aux États-Unis. Inspirés également par le théoricien le plus en vogue de l'art de la pêche à la ligne, Izaak Walton (1593-1663), ces livres exposés en vitrine aujourd'hui ont offert jadis des « rêveries » partagées de plus en plus par des pêcheurs passionnés et désireux de mieux comprendre l'histoire de la pêche sportive. Tirés de la prestigieuse collection de l'ethnologue Richard Gauthier (dont 525 titres anciens et récents d'ouvrages, catalogues, revues et brochures sur la pêche), ces livres 
sont devenus eux-mêmes des objets de rêve pour les bibliophiles du domaine et des objets de savoir pour les muséologues qui les exposent aujourd'hui.

Cette invitation au voyage et au rêve, comment ne pas l'associer par ailleurs au lieu mythique et symbolique par excellence de tout pêcheur, le « lac » ? Le visiteur, déjà imprégné par l'atmosphère féérique dans laquelle il baigne dès son entrée, se laisse conduire vers le cœur virtuel de cette exposition par de belles maquettes miniatures de bateaux et de train, ou mieux encore par ce canot de cèdre dont la pince pointe vers un grand espace multimédia tout en rondeur. Ne règne ici aucun objet, mais plutôt un concept : se raccorder à la nature à travers la contemplation de ses quatre saisons, au rythme des jours et des nuits, au milieu d'un immense paysage circulaire au sein duquel on contemple les entremêlements séculaires des pêcheurs, de la faune et de la flore. Ici se condense l'expérience du pêcheur dans son contact intime avec son environnement, par la pratique de son sport et des techniques qu'il a développées au fil du temps pour en améliorer les performances. Signée Graphics eMotion, cette expérience sensorielle multimédia de la vie au grand air, incarnée par le lac, repose aussi sur l'idée d'éprouver les bienfaits de la nature vierge à travers l'art de la pêche, élevé au rang d'un trait culturel distinctif, condensé dans cette célèbre maxime latine qui en épure le sens en le ciselant à l'extrême : piscator non solum piscatur / Le pêcheur ne fait pas que pêcher : il est un communiant dont l'âme s'extasie devant les beautés de la nature !

Les battements de la vie sauvage rythment nos pas vers le cœur de la collection muséale en explorant le troisième thème de cette exposition, portant sur les sortes de pêche. La sélection d'environ 350 objets qui l'illustrent puise essentiellement dans les collections Muriel Fitzsimmons, Richard Gauthier, Denise Higgins, Réjean Pétrin et Errol Trachy acquises par le Musée à partir de la décennie 2000. À elles seules, les collections Réjean Pétrin (2004) et Richard Gauthier (2012) ont enrichi l'institution muséale d'environ 1800 pièces. La collection nationale du MCQ compte aujourd'hui plus de 2000 objets représentatifs des différents aspects de la pêche sportive en eau douce pratiquée sur le territoire du Québec : pêche à la mouche, au lancer lourd, au lancer léger, pêche urbaine ou pêche blanche. Elle couvre près de 150 ans de pratique du sport. Elle souligne aussi bien le raffinement des objets de pêche en usage par l'élite, de la formation des premiers clubs privés dans les années 1880 à leur fermeture dans les années 1970, que ces nouveautés technologiques issues de la période de démocratisation de ce loisir après la Seconde Guerre mondiale, tels le lancer léger et son fameux moulinet Mitchell de provenance française, qui feront florès jusqu'à nos jours. Cannes, moulinets, coffres, paniers, épuisettes, dandinettes et leurres sont les vedettes de cette riche section de l'exposition. À l'ombre d'une forêt de cannes et de moulinets exposés dans des vitrines cylindriques comme autant d'épinettes noires se dressant sur les rives d'un lac, des îlots thématiques regroupent un matériel de pointe en phase avec la technique à l'honneur. Les séquences d'objets se déclinent selon des conjugaisons singulières : ici saumon-mouches-guides-clubs privés-pêche de l'élite ; là, affichettes de la bière Molson-lancer léger-moteur Evinrude-combat pour l'accès au loisir de la pêche, parlent aussi d'un parcours parfois trouble. Par la magie des vidéos d'ambiance fignolées par la firme réputée Hooké, la pêche à la ligne, déclinée sous toutes ses façons au sein de chacun de ces îlots, trouve malgré 
tout son aboutissement dans l'actualité filmique du jour et la ferveur toujours vibrante et renouvelée de ses aficionados contemporains.

Arrêtons-nous l'espace d'un instant pour nous pencher de plus près sur cette pratique - la pêche à la mouche -, dont les objets représentés reflètent la richesse de la clientèle des clubs privés qui ont fait fureur au Québec pendant une bonne centaine d'années. L' «Acte pour faciliter la formation en cette province de clubs pour la protection de la faune et du gibier », adopté en 1885 et généreusement prêté par la Bibliothèque de l'Assemblée nationale pour les fins de cette exposition, rappelle une histoire qui s'est transformée en guerre des saumons et des truites à l'orée des années 1970, mais qui avait pour objectif à l'origine de permettre à l'État de protéger plus efficacement une faune en grave danger d'extinction. Plus qu'ailleurs dans cette exposition, l'équipement de qualité des sportsmen, en majorité des anglo-américains, souvent propriétaires de droits exclusifs de pêche, force encore l'admiration devant leur beauté. Les étuis à canne en cuir souple, gravés au nom de Farrow Stewart Stranaham, de St. Albans au Vermont, voisinent celui du frère du premier ministre du temps, Charles-Edmond Taschereau, frappé en 1896 de ses initiales CET, non sans rappeler au passage que politique et affaires pouvaient se conjuguer selon les intérêts des parties et les besoins du moment. Le coffre à mouches de Joseph Clovis Arseneault, la sculpture en taille réelle par Jean-Paul Dubé du légendaire guide Richard Adams, le registre des prises (1906-1921) du renommé « Matamajaw Salmon Club » de Causapscal tracent les contours d'une ère qui a vu prospérer sur les rives de la Cascapédia, de la Matapédia et de la Restigouche des camps à l'architecture typique. Ils nous renvoient en même temps aux propos d'un braconnier de la région de Rimouski, rapportés par Paul-Louis Martin dans son article, qui conclut par une boutade qu'il était temps aussi que ces poissons-là « apprennent à parler français »!

Un magnifique Mur de truites, une fresque murale comptant vingt-cinq poissons peints pour la plupart par Robert Wakeham Pilot (1898-1967), résume admirablement un demi-siècle de pêche sportive au Murray Fishing Club sur la rivière Malbaie. Cette œuvre remarquable, restaurée par les soins du Centre de conservation du Québec en 2006 (voir Stéphane Doyon, «Un mur de belles prises », Rabaska 14, p. 132-134), symbolise en même temps tous ces à-côtés de la pêche, faits de retrouvailles joyeuses, de repas animés, de feux de camp à la belle étoile, de chasse aux petits ménés sur le bord du quai, d'histoires de pêche mi-vérité mi-mensonge. Avec la collaboration du Musée de la mémoire vivante, le MCQ a eu l'heureuse idée de parsemer un parcours sans faute par des histoires de pêche, vrais récits collectés dans les principales régions du Québec. Qui dit vrai, qui dit faux ? racontent d'habiles « documenteurs » en fin de course, qui mêlent astucieusement la fiction aux histoires de pêches qui « sonnent vraies » aux oreilles des visiteurs. Qui eut crû que perchaude ou truite étaient aussi des animaux à fourrure? Et pourtant...Une application développée par le Musée, « Pars à la pêche » intéressera petits et grands dans la poursuite de leurs poissons favoris tout au long de leur chemin. Un dernier arrêt sur le quai du lac virtuel clôt enfin une exposition qui aura permis aux visiteurs de marcher dans les traces de ceux qui ont écrit cette très belle histoire de la pêche au Québec.

Cette exposition temporaire, en cours présentement, est présentée par la SÉPAQ et par la Fondation de la faune du Québec, en collaboration avec de nombreux 
partenaires. Elle se termine le 6 septembre 2021. L'idée au point de départ en revient au conservateur retraité Christian Denis, à qui l'on doit aussi le formidable développement de la collection du Musée en matière de pêche. L'équipe de projet qui l'a conçue réunit la chargée de projet Anouk Gingras, la conservatrice Lydia Bouchard et le designer Charles Saint-Gelais. Catherine Gaumond avait entamé les recherches sur le sujet en confiant un mandat à cet effet à l'ethnologue et historien Paul-Louis Martin. On trouvera sur place un panneau explicatif qui rend à une multitude de collaborateurs les crédits qui leur reviennent mais que ce compte rendu ne pouvait énumérer dans le détail. Je tiens à remercier cependant la conservatrice Lydia Bouchard qui m'a fait visiter l'exposition pour les fins de cet article. 